\title{
Proximity and anomalous field-effect characteristics in double-wall carbon nanotubes
}

\author{
Jie Lu, Sun Yin, Z. Z. Sun, and X. R. Wang* \\ Physics Department, The Hong Kong University of Science and Technology, Clear Water Bay, Hong Kong SAR, China \\ L. M. Peng \\ Key Laboratory for the Physics and Chemistry of Nanodevices and \\ Department of Electronics, Peking University, Beijing 100871, China
}

(Dated: June 28, 2021)

\begin{abstract}
Proximity effect on field-effect characteristic (FEC) in double-wall carbon nanotubes (DWCNTs) is investigated. In a semiconductor-metal (S-M) DWCNT, the penetration of electron wavefunctions in the metallic shell to the semiconducting shell turns the original semiconducting tube into a metal with a non-zero local density of states at the Fermi level. By using a two-band tight-binding model on a ladder of two legs, it is demonstrated that anomalous FEC observed in so-called S-M type DWCNTs can be fully understood by the proximity effect of metallic phases.
\end{abstract}

PACS numbers: 73.63.Fg, 73.63.-b, 73.20.At

Introduction- Carbon nanotubes (CNTs) have attracted much attention in recent years because of their novel properties $\frac{1}{1}$ and potential applications in devices ${ }^{2}$ and wiring 3 in nano and molecule electronics. The recent advance in technology allows the fabrication of both single wall carbon nanotubes (SWCNTs) and multi-wall carbon nanotubes (MWCNTs) with controllable diameters. Depending on its chirality ${ }^{1}$, a SWCNT can be metallic or semiconducting. Various issues 4.5 have been examined, including electronic transport 6.7 .8 .9 .10 of different types of CNTs.

Very recently, field-effect transistors have been fabricated out of double-wall carbon nanotubes (DWCNTs) which are the simplest MWCNTs. Field-effect characteristic (FEC), which is about how source-drain current $I_{s d}$ depends on gate voltage $V_{G}$, has been measured ${ }^{11}$. Each DWCNT devices can experimentally be classified into one of three groups, corresponding to semiconductor-semiconductor (S-S), metal-metal (M-M) or metal-semiconductor (M-S), and semiconductor-metal (S-M) combinations of two shells of DWCNTs (the first symbol is for the outer shell and the second for the inner one). The S-S and M-M (or M-S) DWCNTs exhibit similar FEC as those of semiconducting and metallic SWCNTs ${ }^{10}$, respectively. However, S-M DWCNT devices show FEC with distinct features from both of its metal and semiconductor counterparts. 1) In the negative $V_{G}$ region, on-off current ratio $\left(I_{o n} / I_{o f f}\right)$ can be as large as $10^{1}$ or even $10^{2}$. This is in contrast with no obvious switching characteristic for metallic tubes or order of $10^{5}$ on-off ratio for semiconducting ones. 2) The gate-voltage dependence of source-drain current is not exponential in transition region from on to off states as one will expect from the orthodox semiconductor physics theory. 3) The transition region is much wider than that of usual semiconductors. 4) For positive $V_{G}$, it seems that free charges in the metallic tube may screen the outer shell from the gate effect, harmful to field-effect transistors.

In this paper, we would like to attribute the anomalous
FEC observed in the so-called S-M DWCNTs to the proximity effect of metallic phase, a general property of waves. A qualitative explanation of the anomalous FEC is presented first. The penetration of electron wavefunctions in the inner metallic tube into the outer semiconducting shell due to the inter-shell coupling generates local electron density of states (DOS) in the semiconducting tube in its original forbidden gap. Thus, new conducting channels are created in the gap, and the semiconducting tube becomes weakly conducting. The picture is verified by modeling a DWCNT by a two-band tight-binding Hamiltonian on a ladder of two legs. The results capture the essential features of the observed FEC.

Picture-Consider a S-M DWCNT under a gate voltage $V_{G}$ schematically shown in Fig 17, a source-drain bias $V_{s d}$ is applied between the two ends of the outer DWCNT shell. The inner and outer tubes are assumed to be metallic and semiconducting, respectively. Their energy band structures are like those of the right and the middle parts of Fig 1b. Without an inter-shell coupling, the system consists of two independent tubes with their own energy bands. In the absence of the gate voltage, the DOS in the outer shell at the Fermi level is zero. Thus, electrons (holes) can move from the source to the drain through either the inner metallic shell by quantum tunneling or the outer-shell conduction (valence) band by thermal activation. Both process contribute to the leakage current in field-effect transistors, and are small at low temperature with $k T \ll E_{g}$. Applying a negative gate voltage, the top of the valence band will be pushed upward (shown by the big arrows in Fig. 11) so that hole conduction will increase exponentially with the gate voltage, leading to the FEC of a usual semiconductor device.

In reality, there is always an inter-shell coupling in a DWCNT. Electron states in two shells are hybridized with each other such that the local DOS of the two shells will be modified (the total DOS may not change much). For example, the local DOS of the outer semiconducting shell in its original energy gap becomes non-zero because inner-shell states in the energy range have certain prob- 
(a)
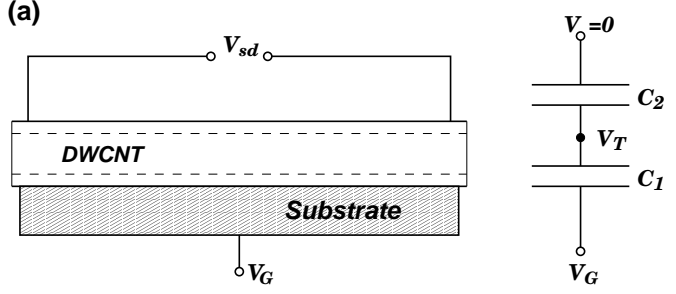

(b)

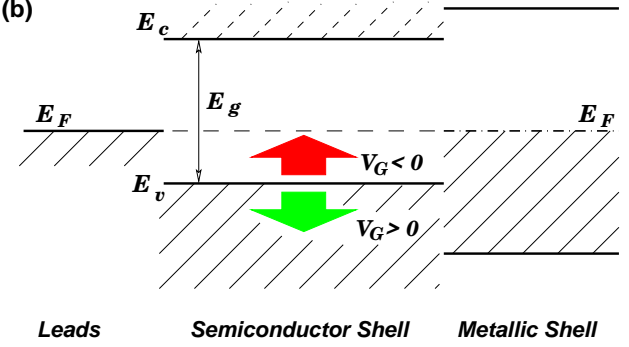

(c)

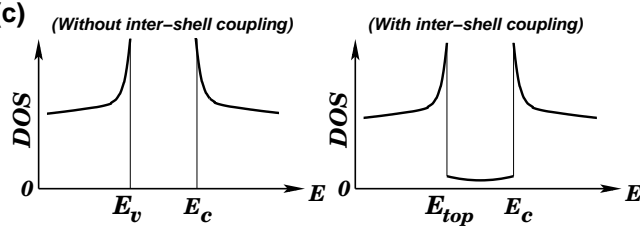

FIG. 1: (a) Schematic experimental set-up of measuring FEC of a DWCNT. $V_{s d}$ and $V_{G}$ are source-drain bias and gate voltage, respectively. $C_{1}$ and $C_{2}$ are the capacitance between the gate and the DWCNT and that of the DWCNT itself. $V_{T}$ is the potential on the tube. (b) Schematic energy bands of the outer semiconducting shell (middle part), and the conduction band of the inner metallic shell (right part). $E_{F}$ is the Fermi level of the metallic leads. $E_{c}\left(E_{v}\right)$ is the bottom (top) of the conduction (valence) band and $E_{g}$ is the energy gap. The big arrows indicate the moving directions of $E_{v}$ under $V_{G}$. (c) The DOS in the outer shell without and with an inter-shell coupling which leads to a non-zero DOS in the gap region.

ability to appear on the outer shell. The penetration of electron wavefunctions from the metallic shell into the semiconducting shell is called the proximity effect. For comparison, the DOS of the outer shell with and without inter-shell coupling are illustratively plotted in Fig 11. Its value in the original gap region is sensitive to the energy barrier $E-E_{v}$ for the electrons in the inner shell, where $E$ and $E_{v}$ are the electron energy and top of the valence band, respectively. This barrier can be controlled by the gate voltage $V_{G}$ because $E_{v}$ is pushed upward under a negative $V_{G}$, resulting in the decrease in the barrier and increase in the local DOS of the outer shell.

The appearance of DOS in the outer shell in the energy gap creates new electron conducting channels. At a small $V_{G}$ and under a small $V_{s d}$, all current can be carried by the local states in the outer shell, and there is no need to use the inner shell although the DOS there near the Fermi level is much greater than that in the outer one. This feature has been observed in MWCNT ${ }^{4,12,13}$. The proximity effect, which becomes stronger and stronger as the gate voltage is more and more negative, turns the semiconducting outer shell into a weak metal. The gate-voltage dependence of this metallicity leads to the anomalous FEC in S-M DWCNTs. Since the origin of the anomaly is from the emergence of DOS in the outer shell in its gap region, the proximity effect is important only when the Fermi level lies in the gap, i.e. $E_{v}<E_{F}<E_{c}$. This explains the relative wide transition region, as well as the non-exponential dependence of $I_{s d}$ on $V_{G}$.

Model-To put the above picture on a firm quantitative analysis, a S-M DWCNT is modeled by a two-leg ladder as shown in Fig 2 One leg, say leg $A$, is used to model the inner metallic shell, and the other, $\operatorname{leg} B$, is for the outer semiconducting shell. If the source-drain bias is not very large, then there is only one band from each shell relevant to electronic transport. Following the experimental situation $^{11}$, it is assumed that the semiconducting shell is originally $p$-doped so that its Fermi level is near the top of the valence band. However, it is trivial to generalize the following discussion to an $n$-doped tube where the conduction band will be considered and the band bottom is slightly above the Fermi level. A tight-binding model is defined on the ladder with $t_{A}$ and $t_{B}$ (order of the shell bandwidths) being the hopping coefficients on leg $A$ and $B$, respectively. The inter-shell coupling is described by inter-chain hopping coefficient $t^{\prime}$. The Hamiltonian is

$H=\sum_{\alpha, i}\left(\frac{1}{2} \varepsilon_{\alpha} c_{\alpha, i}^{\dagger} c_{\alpha, i}+t_{\alpha} c_{\alpha, i}^{\dagger} c_{\alpha, i+1}\right)+\sum_{i} t^{\prime} c_{A, i}^{\dagger} c_{B, i}+$ h.c.

where $\alpha=A, B$ labels the legs, $\varepsilon_{\alpha}$ are the on-site energies. $c_{\alpha, i}^{\dagger}$ and $c_{\alpha, i}$ are the creation and annihilation operators at site $i$ of leg $\alpha$, respectively. The Hamiltonian has two branches of eigen-energies,

$$
\begin{aligned}
E_{ \pm, k}= & \frac{1}{2}\left(\varepsilon_{A}+\varepsilon_{B}\right)+\left(t_{A}+t_{B}\right) \cos k \\
& \pm \frac{1}{2} \sqrt{\left[\left(\varepsilon_{A}-\varepsilon_{B}\right)+2\left(t_{A}-t_{B}\right) \cos k\right]^{2}+4\left|t^{\prime}\right|^{2}}
\end{aligned}
$$

Without inter-chain coupling, i.e. $t^{\prime}=0$, the two branches correspond to two independent bands (width $4 t_{A}$ and $\left.4 t_{B}\right)$ centered at $\varepsilon_{A}$ and $\varepsilon_{B}$, respectively. In general, $\varepsilon_{A}$ and $\varepsilon_{B}$ depend on the gate voltage. However, $\varepsilon_{A}$ is less sensitive to $V_{G}$ because DOS in the inner shell at the Fermi level is large, and any substantial change of $\varepsilon_{A}$ results in a great accumulation of charge in the shell which will generate a large electric potential. This is also often called Fermi level pining (at large DOS). Therefore, the gate voltage applies mainly on the outer shell, and it is reasonable to assume a constant $\varepsilon_{A}$ and $\varepsilon_{B}=U_{0}-e \beta V_{G}$, where $U_{0}$ is the initial on-site energy and $\beta=C_{1} /\left(C_{1}+C_{2}\right)$ relates to the mutual capacitance $C_{1}$ between the substrate and the DWCNT and the selfcapacitance $C_{2}$ of DWCNT as shown in Fig. 17. For a given DWCNT (fixed $C_{2}$ ), larger $C_{1}$ means larger $\beta$. $\beta$ increases as the substrate thickness decreases because $C_{1}$ becomes bigger. This explains why $I_{s d}$ under the same $V_{s d}$ is bigger with a thinner $\mathrm{SiO}_{2}$ layer ${ }^{11}$ since a thin substrate is more effective in applying a gate voltage. Without losing generality, the Fermi level of the source 
lead is set to zero. Model parameters are chosen so that $E=0$ is inside the energy band of leg-A but outside that of leg-B at $V_{G}=0$.



FIG. 2: A S-M DWCNT (top) is modeled by a two-leg ladder (bottom) on which a tight-binding model is defined. $t_{A}\left(t_{B}\right)$ is the hopping coefficient in leg $\mathrm{A}(\mathrm{B})$, and $t^{\prime}$ is the inter-chain hopping coefficient.

As it was mentioned early, the important quantity is the DOS in leg $B$ which can be evaluated by

$$
g_{B}(E)=\frac{1}{2 \pi} \sum_{\lambda= \pm, k}\left|\frac{\partial E_{\lambda, k}}{\partial k}\right|^{-1} P_{\lambda, k, B} \delta\left(E_{\lambda, k}-E\right),
$$

where $P_{\lambda, k, B} \equiv \sum_{i_{B}}\left|\psi_{\lambda, k}\left(i_{B}\right)\right|^{2}$ is the probability of finding an electron in leg $B$ when it is in eigenstate $\psi_{\lambda, k}(x)$.

Results and discussions- To have a better picture about how large the proximity effect is and to make a quantitative comparison with experimental data, the model parameters will be set as follows: $t_{A}=0.75 \mathrm{eV}$; $t_{B}=0.3 \mathrm{eV} ; \varepsilon_{A}=0 \mathrm{eV} ; U_{0}=-.65 \mathrm{eV} ; t^{\prime}=0.35 \mathrm{eV}^{5.14}$, corresponding to a typical subband width of $1 \mathrm{eV} \sim 4 \mathrm{eV}$ of $\mathrm{CNTs}^{5.14}$, and the top of the valence band of the outer shell being about $0.05 \mathrm{eV}$ below $E_{F}$ at $V_{G}=0$. Thus, $E_{F}=0$ is inside the band of leg-A. The inset of Fig [3] is $g_{B}(E)$ as a function of energy $E($ in $e V)$ at $V_{G}=0$. The DOS in the energy range of $-1.75 \mathrm{eV}<E<-0.13 \mathrm{eV}$ is the sum of the contribution from '-' branch of (2), a 1-D tight-binding model like, plus the proximity effect contribution from '+'-branch of (2) in the range of $-1 e V \leq E \leq-0.13 e V$. Its typical value is of order of $1 /(e \bar{V} \cdot$ site $)$. The non-zero DOS outside of this energy range is completely from the proximity effect of ' + 'branch of (2). The spikes are the van Hove singularities of the 1-D tight-binding model, a model-dependent feature.

Fig[3] is the local DOS $g_{B}\left(E_{F}\right)$ in leg-B at the Fermi level as a function of gate voltage $V_{G}$ (in volts $V$ ). The model parameters are the same as those in the inset of Fig. 3] and $\varepsilon_{B}=U_{0}-e V_{G}(\beta=1)$ is used for simplicity. For large $V_{G}$, the Fermi level is deep inside the original energy gap, and the value of $g_{B}\left(E_{F}\right)$ is small, order of $10^{-3}(\mathrm{eV} \cdot \mathrm{site})^{-1}$. The Fermi level approaches the top of valence band when $V_{G}$ becomes negative. Near $V_{G}=$ $-0.13 \mathrm{~V}$, the DOS is order of $10^{-1}(\mathrm{eV} \cdot \mathrm{site})^{-1}$, a order of $10^{2}$ change in electronic conduction as $V_{G}$ varies.

Under a small source-drain bias $V_{s d}$, the current $I_{s d}$ is proportional to the local DOS on the outer shell at the

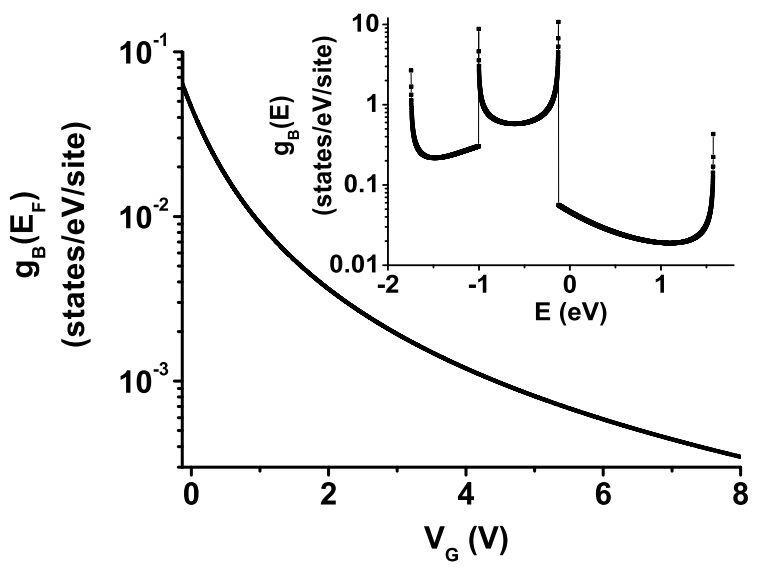

FIG. 3: The logarithm of $g_{B}\left(E_{F}\right)$ vs. gate voltage $V_{G}$ when $\varepsilon_{B}=U_{0}-e V_{G}$ is used. Inset: The logarithm of $g_{B}(E)$ vs. $E$ at $V_{G}=0$.

Fermi level. Fig. 4 is the fitting curve of experimental FEC at temperature $(292 K)^{11}$ by $g_{B}\left(E_{F}\right)$ for $V_{G} \geq-5 V$, where proximity effect dominates the electron transport process. Here, it is interpreted that the top of the valence band touches the Fermi level at $V_{G}=-5 \mathrm{~V}$ in the experiment ${ }^{11}$. Thus both of the states from the proximity effect and from the orginal valence band particpated in the transport. This is why a reflexion point occurs in the experimental $I_{s d}$ curve at $V_{G}=-5 \mathrm{~V}$, and the present theory should only compare with the data for $V_{G} \geq-5 \mathrm{~V}$. In the figure, $\varepsilon_{A}-\varepsilon_{B}=-U_{0}+\beta e V_{G}$ and $I_{s d}=\eta g_{B}$ are used. The fitting parameters are $t_{A}=0.95 \mathrm{eV}$; $t_{B}=0.44 \mathrm{eV} ; t^{\prime}=0.42 \mathrm{eV} ; \varepsilon_{A}=0.11 ; U_{0}=-1.05 \mathrm{eV}$; $\beta=0.025$; and $\eta=3.1 \mu \mathrm{A} \cdot \mathrm{eV}$. These values are reasonable for CNTs, and it is surprising that these values can be obtained by fitting the experiments to such a simple model. The fitting is not bad for $I_{s d} \geq 60 \mathrm{nA}$. The deviation for $I_{s d}<60 \mathrm{nA}$ can be attributed to the leakage current or the gate-voltage dependence of $\beta$ due to screening effect. It is not difficult to understand the deviation because the proximity effect does not take into account the leakage current and the screening effect. The leakage current is very sensitive to the temperature as what have been observed in experiment ${ }^{11}$.

$I_{o n} / I_{o f f}$ is sensitive to the temperature. At zero temperature, $I_{\text {off }}$ comes completely from the proximity effect through the appearance of the local DOS in the outer shell in the gap region. Increase the temperature, an extra current from electrons (holes) in the conduction (valence) band of the outer shell is added to $I_{o f f}$. This extra current could be comparable or even bigger than that of the proximity effect at high enough temperature. This extra leakage current will also add to $I_{o n}$, but it will be less important because of relative large current of $I_{s d}$ in the on-state. In general, this ratio decreases with the increase of the temperature, exactly as what was found in experiments 11 . 


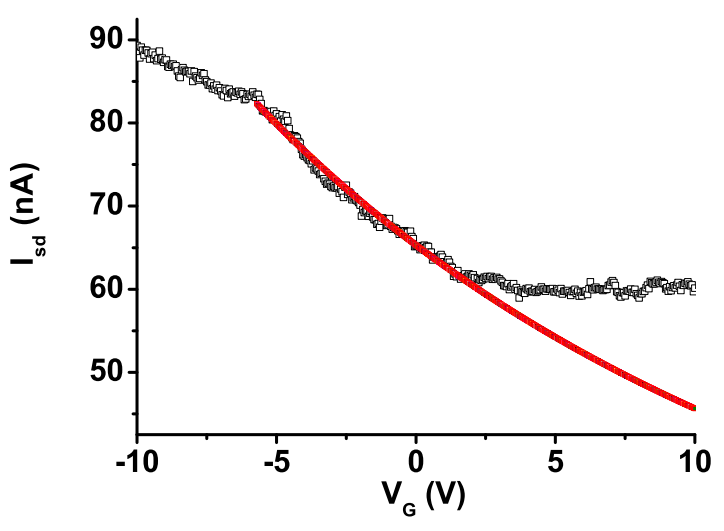

FIG. 4: Fitting between the analytical form (solid line) of the local DOS in the outer (semiconducting) shell at Fermi level and the experimental data (Ref. 11) at $292 K$. The saturated $I_{s d}$ (around 60nA) is interpreted as the leakage current. Good fit indicates applicability of the simple model.

It should be emphasized that the anomalous FEC observed in S-M DWCNTs is not due to change of holes (or electrons in the conduction band of semiconductors) as that of usual semiconductor field-effect transistors under a gate voltage. Rather, it is due to change of DOS originated from the proximity effect at the Fermi level. Although the detail atomic and energy-band structures of DWCNT are neglected, the present model reveals the essential physics of the anomalous FEC of S-M DWCNTs. It implies that the anomaly is a common feature of all S-M layered structures, not necessary for CNTs only. This is because the proximity effect is a general property of wave nature of particles, independent of system details. The proximity effect is not new. It is an important effect in superconductivity that has received intensive investigation. The Josephson tunneling is one of its manifestations. The difference of the proximity effect in superconductors and in DWCNTs is that the particles are Cooper pairs in the former and electrons in the latter.

Electron-electron (e-e) interactions were neglected in this study. In general, e-e interactions are important in low temperature physics and for the narrow bands. For CNTs, the band width is quite large (order of $1 \sim 4 \mathrm{eV}$ ) so that the net e-e interaction may be reduced through screening. So far, most physics quantities measured in experiments have not shown the importance of the e-e interactions. Thus, it is safe to neglect the interaction for the anomalous FEC which was measured at $292 \mathrm{~K}$. However, the interaction may be very important for very low temperature physics and very small wires where the Luttinger liquid (non Fermi liquid) behavior will dominate eventually.

Conclusion - The anomalous FECs in so-called S-M DWCNTs is explained in terms of one-electron energy band structure. The essential physics is the proximity effect of electrons: The proximity effect creates local DOS in the gap region, resulting in new conduction channels in the semiconducting outer tube. The proximity effect is stronger and stronger when the Fermi level approaches the top (bottom) of the valence (conduction) band of the outer p-doped (n-doped) semiconducting tube. As a result, the semiconducting outer tube becomes more and more metallic.

Acknowledgments-This work is supported by UGC, Hong Kong, through RGC CERG grants (No. HKUST6067/02P; 603904). LMP is supported by NSF of China (Grant No. 10434010).
* To whom correspondence should be addressed. Electronic address: phxwan@ust.hk

1 M.S. Dresselhaus, G. Dresselhaus, and P.C. Eklund, Science of Fullerenes and carbon Nanotubes, Academic Press, London, 1995; R. Saito, G. Dresselhaus, and M.S. Dresselhaus, Physical Properties of Carbon Nanotubes, Imperial College Press, London, 1998.

2 L. Chico, V.H. Crespi, L.X. Benedict, S.G. Louie, and M.L. Cohen, Phys. Rev. Lett. 76, 971 (1996); C.T. White, and T.N. Todorov, Nature 393, 240 (1998).

3 S.J. Tans, M.H. Devoret, H.J. Dai, A. Thess, R.E. Smalley, L.J. Geerligs, and C. Dekker, Nature 386, 474 (1997); Z. Yao, H.W.C. Postma, L. Balents, and C. Dekker, Nature 402, 273 (1999).

${ }^{4}$ S. Sanvito, Y.-K. Kwon, D. Tománek, and C.J. Lambert, Phys. Rev. Lett. 84, 1974 (2000).

5 S. Roche, F. Triozon, A. Rubio, and D. Mayou, Phys. Rev. B 64, 121401 (2001); Y. Miyamoto, S. Saito, and D. Tománek, Phys. Rev. B 65, 041402 (2001); S. Uryu, Phys. Rev. B 69, 075402 (2004).

6 A. Javey, J. Guo, Q. Wang, M. Lundstrom, H.J. Dai, Na- ture 424, 654 (2003).

7 R. Martel, T. Schimidt, H.R. Shea, T. Hertel, and Ph. Avouris, Appl. Phys. Lett. 73, 2447 (1998).

8 T. Shimada, T. Sugai, Y. Ohno, S. Kishimoto, T. Mizutani H. Yoshida, T. Okazaki, and H. Shinohara, Appl. Phys. Lett. 84, 2412 (2004).

9 S. Wang and M. Grifoni, Phys. Rev. Lett. 95, 266802 (2005).

10 S.J. Tans, A.R.M. Verschueren, and C. Dekker, Nature 393, 49 (1998).

11 S. Wang, X.L. Liang, Q. Chen, Z.Y. Zhang, and L.-M. Peng, J. Phys. Chem. B 109, 17361 (2005).

12 S. Frank, P. Poncharal, Z.L. Wang, and W.A. de Heer, Science 280, 1744 (1998)

13 A. Urbina, I.Echeverría, A. Pérez-Garrido, A. DíazSánchez, and J. Abellán, Phys. Rev. Lett. 90, 106603 (2003).

14 J.C. Charlier, and J.P. Michenaud, Phys. Rev. Lett. 70, 1858 (1993); R. Saito, G. Dresselhaus, and M.S. Dresselhaus, J. Appl. Phys. 73, 494 (1993). 